\title{
Best Practices in Adolescent and Young Adult Patients with Acute Lymphoblastic Leukemia: A Focus on Asparaginase
}

\author{
Nicolas Boissel, MD, PhD, and Leonard S. Sender, MD 2,3,4
}

The inclusion of asparaginase in chemotherapy regimens to treat acute lymphoblastic leukemia (ALL) has had a positive impact on survival in pediatric patients. Historically, asparaginase has been excluded from most treatment protocols for adolescent and young adult (AYA) patients because of perceived toxicity in this population, and this is believed to have contributed to poorer outcomes in these patients. However, retrospective analyses over the past 12 years have shown that 2-, 5-, and 7-year overall survival of AYA patients is significantly improved with pediatric versus adult protocols. The addition of asparaginase to adult protocols yielded high rates of first remission and improved survival. However, long-term survival remains lower compared with what has been seen in pediatrics. The notion that asparaginase is poorly tolerated by AYA patients has been challenged in multiple studies. In some, but not all, studies, the incidences of hepatic and pancreatic toxicities were higher in AYA patients, whereas the rates of hypersensitivity reactions did not appear to differ with age. There is an increased risk of venous thromboembolic events, and management with anti-coagulation therapy is recommended. Overall, the risk of therapy-related mortality is low. Together, this suggests that high-intensity pediatric protocols offer an effective and tolerable approach to treating ALL in the AYA population.

Keywords: acute lymphoblastic leukemia, asparaginase, young adult, adolescent

A CUTE LYMPHOBLASTIC LEUKEMIA (ALL) is a heterogenic disease that disproportionately affects children between the ages of 2 and 10 years. ${ }^{1-3}$ Roughly 6000 new cases of ALL are diagnosed each year in the United States alone. ${ }^{2,4}$ Substantial advancements have been made in crafting effective multiagent pediatric chemotherapeutic protocols, raising overall survival (OS) rates for pediatric patients to nearly $90 \% .^{5,6}$ Unfortunately, there has been less success in achieving these same improvements in adolescent and young adult (AYA) patients diagnosed with ALL. ${ }^{7}$ In contrast to the high survival rates in pediatric patients, 5-year survival rates for adolescents (aged 15-19 years) and young adults (aged 20-29 years) diagnosed with ALL are approximately $61.1 \%$ and $44.8 \%$, respectively (1973-2004 limited use database of the Surveillance, Epidemiology, and End Results Program of the U.S. National Cancer Institute issued in April 2007). ${ }^{7}$

Poor outcomes in AYA patients diagnosed with ALL likely result from a number of factors. AYA patients display a greater incidence of adverse prognostic indicators, including an increased prevalence of adverse genetic abnormalities, compared with younger patients. ${ }^{8,9}$ Several socioeconomic factors, such as reduced enrollment in clinical trials, possibly lower compliance rates, and increased delays in treatment initiation, may also contribute to the poor outcomes seen in the AYA population. ${ }^{10}$ Arguably, one of the most important factors in the disproportionate survival between pediatric and AYA patients are the chemotherapy protocols historically used to treat these two groups of patients. Most pediatric ALL protocols follow the Berlin-Frankfurt-Munster (BFM) model with a heavy reliance on nonmyelosupressive agents (vincristine, steroids, and asparaginase). ${ }^{11}$ Adult protocols were for a long time characterized by a greater use of myelosupressive agents (including cyclophosphamide and anthracyclines) with little or no asparaginase, as the enzyme was perceived to be prohibitively toxic in adults. ${ }^{12}$ In fact, one widely used adult ALL protocol, hyper-CVAD, includes no asparaginase. ${ }^{13}$ Recently, a wealth of clinical evidence has emerged challenging the differential treatment of pediatric

\footnotetext{
${ }^{1}$ Department of Adult Hematology, Saint-Louis Hospital, University of Paris, Paris, France.

${ }^{2}$ Department of Epidemiology; ${ }^{3}$ Chao Family Comprehensive Cancer Center; University of California, Irvine, Irvine, California.

${ }^{4}$ Hyundai Cancer Institute, CHOC Children's Hospital, Orange, California.
}

(C) Nicolas Boissel and Leonard S. Sender, 2015; Published by Mary Ann Liebert, Inc. This Open Access article is distributed under the terms of the Creative Commons License (http://creativecommons.org/licenses/by/4.0), which permits unrestricted use, distribution, and reproduction in any medium, provided the original work is properly credited. 
and AYA patients with ALL. ${ }^{14-21}$ Retrospective studies report that high-intensity pediatric protocols are both feasible and tolerable in patients aged $\geq 15$ years and that the use of these protocols is associated with substantially improved long-term survival compared with commonly used adult protocols. ${ }^{14,22-24}$ This review will summarize the relevant differences between pediatric and AYA leukemia biology and treatment, highlighting the rationale for treating adult patients according to pediatric protocols. The use of asparaginase, a near-universal component of pediatric ALL regimens, will be reviewed in detail.

\section{Disease Biology}

There are a number of biological factors that contribute to low cure rates in AYA patients. T-cell ALL is known to be associated with poor outcomes and is seen in $20-25 \%$ of adult cases of ALL compared with $15 \%$ in children. ${ }^{15,25}$ Furthermore, early T-cell precursor leukemia is a particularly high-risk subtype of T-cell ALL found in approximately $15 \%$ of T-cell ALL. This subtype has been characterized by a high risk of treatment failure and poor prognosis in patients. ${ }^{26,27}$ However, recent results in larger patient populations have shown noninferior outcomes in patients with T-cell and early T-cell precursor ALL. ${ }^{28}$ Several chromosomal abnormalities associated with negative outcomes are more commonly observed in adult patients relative to pediatric patients with ALL. ${ }^{29}$ The incidence of Philadelphia chromosome positive (Ph1) ALL, strongly associated with poor outcomes, is one of the most common cytogenetic abnormalities in adult ALL. ${ }^{30} \mathrm{Ph} 1 \mathrm{ALL}$ is found in 15-20\% of patients aged 25-35 years compared with $<3 \%$ of patients aged $<18$ years. ${ }^{23,30,31}$

Further compounding the problem, genetic alterations associated with positive prognosis occur less often in AYA patients. High hyperdiploidy, associated with favorable prognosis, occurs in 25-30\% of pediatric patients with ALL, but only in $20 \%$ of adolescents and $10 \%$ of young adult patients. ${ }^{23}$ Similarly, the TEL-AML1 fusion gene occurs in approximately $25 \%$ of pediatric cases compared with just $3 \%$ of adults with ALL. ${ }^{32}$ Other adverse prognostic features, such as intrachromosomal amplification of chromosome 21, MLL translocations, and IGH@ translocations, have been shown to occur more frequently in the AYA population. ${ }^{26,33,34} \mathrm{Ph} 1$-like ALL is a subtype of B-cell ALL with similar patterns of gene expression to Ph1 ALL, but does not express the BCR-ABL1 fusion protein. ${ }^{35,36} \mathrm{Ph} 1$-like ALL has been reported in $27 \%$ of B-cell ALL patients between 21 and 39 years of age, compared with $10 \%$ of patients between 1 and 9 years of age. ${ }^{36}$ This subtype has been associated with a resistance to asparaginase and daunorubicin, ${ }^{35}$ higher MRD levels after induction, ${ }^{37}$ and a higher risk of relapse in the AYA population. ${ }^{36}$

Changes in drug resistance may also occur with age, resulting in reduced benefits from chemotherapy and poor outcomes in AYA patients. Leukemic cells from patients aged $>10$ years were significantly more resistant to prednisolone, dexamethasone, asparaginase, idarubicin, and 6mercaptopurine compared with leukemic cells from children aged 1.5-10 years. ${ }^{38}$ Additionally, genetic factors specific to genes regulating the immune system, found in both pediatric and adult patients, have recently been shown to increase a patient's risk for developing an allergic reaction to asparaginase. ${ }^{39}$ Metabolism of certain chemotherapeutic drugs may also differ in adult patients, possibly increasing the incidence of drug-related toxicities or decreasing the effectiveness of critical chemotherapeutic agents, such as asparaginase or dexamethasone. ${ }^{40}$

\section{Treatment of AYA Patients with ALL}

There are important differences in how ALL is treated in pediatric and AYA patients that may contribute to the poor outcomes observed in these patients. AYA patients are less likely to be enrolled in clinical trials. Clinical trials help to maintain standardization of care and may be an important factor toward ensuring the effectiveness of treatment. ${ }^{10,41-43}$ One American study found that $<5 \%$ of AYA cancer patients were enrolled in clinical trials compared with $60 \%$ of pediatric patients. ${ }^{44}$ Additionally, children with ALL are generally cared for by a parent or legal guardian who may help maintain compliance throughout the prolonged treatment schedules. Treatment compliance is often problematic in AYA patients who may require substantial support to cope with treatment-related toxicities. ${ }^{45-47}$ Bhatia et al. evaluated adherence to mercaptopurine treatment schedules in 327 patients diagnosed with ALL. ${ }^{43}$ The results showed significantly lower adherence over a 6-month period in patients aged $\geq 12$ years compared with younger patients $(85.8 \%$ vs. $93.1 \%, p<0.001)$. Investigators also found a higher risk of relapse in patients with lower adherence rates, highlighting the importance of high treatment adherence. ${ }^{47}$

While socioeconomic factors may contribute to the poor outcomes seen in AYA patients, likely the most critical-and most readily addressable-difference between pediatric and AYA treatment of ALL is the type of protocol used to treat each patient population. Pediatric protocols typically contain high doses of vincristine, corticosteroids, and asparaginase. In pediatric trials, adolescents are often treated as high-risk patients and are given a more intensified chemotherapy schedule. The treatment of AYA patients typically includes the use of allogeneic stem cell transplantation (SCT), a procedure associated with significant adverse events (AEs) and with a transplantrelated mortality rate up to $20 \%$. Pediatric protocols restrict SCT to a minority of patients with specific high-risk features. ${ }^{48,49}$

During the past decade, a number of retrospective trials have evaluated outcomes in AYA patients treated on either adult or pediatric protocols. ${ }^{15-21}$ The results of these studies show a consistent trend toward improved outcomes for AYA patients treated on pediatric ALL protocols compared with similar patients treated on adult ALL protocols (Fig. 1). ${ }^{15,16,18,19,21}$ Boissel et al. compared outcomes in patients aged 15-20 years treated according to the French ALL Cooperative Group (FRALLE)-93 and Leucemie Aiguë Lymphoblastique de l'Adulte (LALA)-94 protocols. ${ }^{15}$ The pediatric FRALLE-93 protocol included significantly more vincristine, steroids, and asparaginase compared with the adult LALA-94 protocol. In FRALLE-93, patients were exposed to up to 20 times more cumulative amounts of asparaginase compared with patients in LALA-94. Asparaginase was incorporated into induction and delayed intensification in FRALLE-93 compared with a 2-day exposure in LALA-94. Investigators found that the patients treated in the pediatric FRALLE-93 trial showed significantly higher complete response (CR, 94\% vs. 83\%), 5-year eventfree survival (EFS; $67 \%$ vs. $41 \%$ ), and OS (78\% vs. $45 \%$ ) compared with patients treated in the adult LALA-94 trial. ${ }^{15}$ 


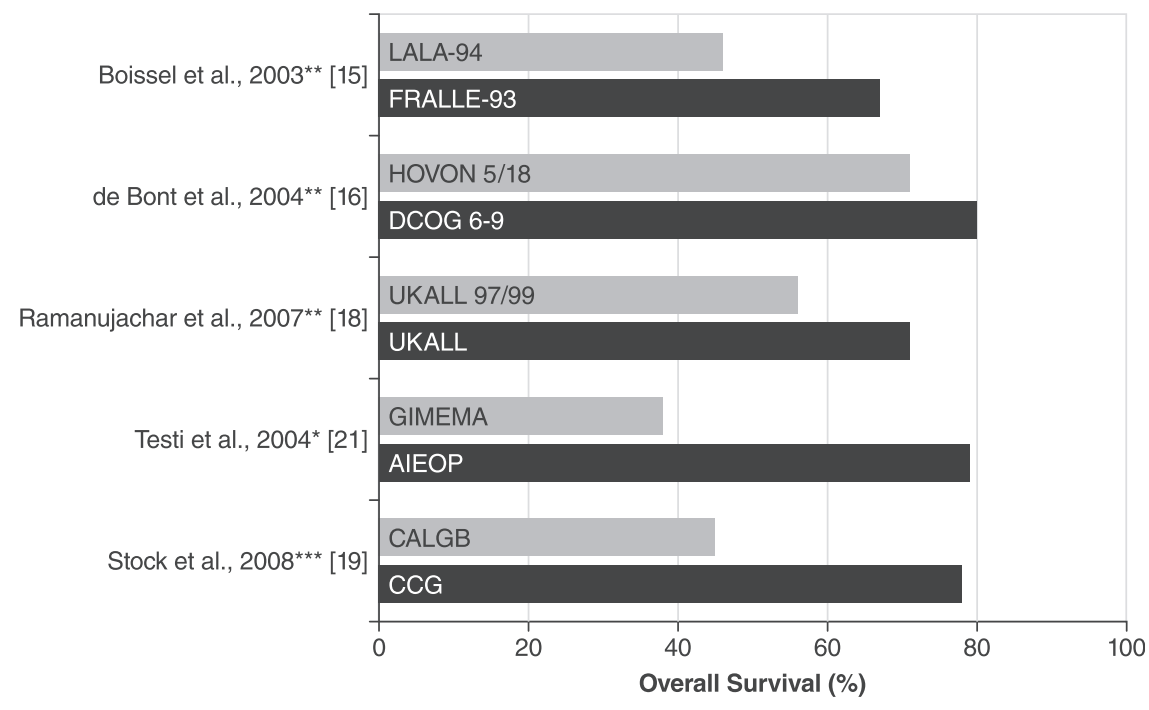

FIG. 1. Retrospective comparison of outcomes in adolescent and young adult (AYA) patients treated on pediatric and adult protocols. *2-year OS; **5-year OS; ***7-year OS. AIEOP, Italian Association of Pediatric Hematology and Oncology; CALGB, Cancer and Leukemia Group B; CCG, Children's Cancer Group; DCOG, Dutch Childhood Oncology Group; FRALLE-93, French ALL Cooperative Group; GIMEMA, Italian Group for Adult Hematologic Diseases; HOVON, Hemato-Oncology Cooperative Study Group; LALA-94, Leucemie Aiguë Lymphoblastique de l'Adulte; OS, overall survival.
The North American Cancer and Acute Leukemia Group B (CALGB) and the Children's Cancer Group (CCG) also retrospectively evaluated patients treated according to adultor pediatric-based protocols. ${ }^{19}$ Overall, 321 AYA patients, aged 16-20 years, were treated on either adult CALGB or pediatric CCG treatment regimens. CALGB trials included a five-drug remission induction regimen (cyclophosphamide, daunorubicin, vincristine, prednisone, and asparaginase). Patients in the CCG trials were treated with either a standard CCG protocol or an augmented regimen that included greater exposure to asparaginase during consolidation, interim maintenance, and delayed intensification. At a 7-year followup, AYA patients in CCG showed EFS of $63 \%$ and OS of $67 \%$ compared with AYA patients in CALGB who showed EFS of $34 \%$ and OS of $46 \%$. Subsequent retrospective reports revealed similar benefits when evaluating adolescent patients treated with pediatric protocols. ${ }^{16-18}$ Taken together, these results show a consistent trend toward better outcomes for young adolescent patients (aged 15-19 years) treated on pediatric regimens with greater cumulative doses of vincristine, steroid, and asparaginase.

Although these results highlight substantial improvements for adolescents treated on pediatric regimens, the patient populations in these retrospective studies were restricted to individuals aged 20 years and younger. Treatment-related AEs associated with asparaginase-containing regimens are generally believed to increase with age. Patients aged 46-60 years showed poor tolerance of asparaginase when compared with patients aged $14-45$ years. ${ }^{50}$ Establishing the feasibility of asparaginase use in the older adult population is currently a critical area of research.

Results of several recent clinical trials suggest that the improved outcomes with pediatric-like treatment protocols can be generalized to the broader AYA population. ${ }^{50-53}$ Storring et al. evaluated 85 BCR-ABL-negative newly diagnosed ALL patients (aged 18-60 years) treated on a modified Dana-Farber Cancer Institute (DFCI) 91-01 Consortium Protocol, which included high-dose weekly asparaginase for 30 weeks during induction. ${ }^{51}$ The CR rate in these adult patients was $89 \%$, and the 5-year OS was $63 \%$. Age was a prognostic factor, with younger adult patients, aged 35 years or younger, showing greater 3-year OS compared with patients older than 35 years of age (83\% and 52\%, respectively). Prolonged asparaginase treatment was generally well tolerated, with only 12 patients failing to receive the complete 30 weeks of scheduled asparaginase therapy during intensification. Interestingly, patients who received at least $80 \%$ of their planned asparaginase therapy showed significantly greater 3-year OS and a lower cumulative risk of relapse compared with patients who were unable to receive $>80 \%$ of their scheduled treatment. Fathi et al. ${ }^{54}$ examined the efficacy in an older population of a protocol derived from a completed DFCI consortium regimen used in younger adults. Pegylated (PEG)-asparaginase was given to 30 patients aged 51-75 years during induction and Cycle 1 of consolidation phases of therapy. A total of 19 of 29 evaluable patients achieved CR. Of the 18 patients who achieved CR after induction therapy, disease-free survival (DFS) at 1 year was $77 \%$; the 1 -year OS of $61 \%$ for evaluable patients with at least 1 year of follow-up was significantly higher than the historical rate of $33 \%$.

The inclusion of asparaginase is potentially a key factor in the achievement of improved long-term outcomes for AYA patients treated on pediatric protocols. Hyper-CVAD is an asparaginase-free chemotherapeutic regimen widely used to treat adults with ALL. ${ }^{13,55-61}$ This regimen consists of two alternating cycles: Cycle A, characterized by fractionated cyclophosphamide, vincristine, doxorubicin, and dexamethasone, and Cycle B, characterized by high-dose methotrexate and cytarabine. ${ }^{13,59} \mathrm{~A}$ number of recent studies have retrospectively compared patients treated on pediatric-inspired and hyper-CVAD. ${ }^{61-63}$ In one study, patients treated on either the CALGB-8811 or hyper-CVAD treatment regimen were retrospectively compared. ${ }^{61}$ As reviewed above, CALGB-8811 included the use of asparaginase, although at a lower cumulative dose than in pediatric-based protocols. The investigators found that the majority of patients on both protocols achieved first remission, with CR rates of $84 \%$ for hyper-CVAD and 74\% for CALGB-8811. However, a lower 5 -year OS rate was seen in patients treated on hyper-CVAD compared with CALGB-8811 (26\% vs. 44\%, respectively). Similarly, a recent report from Alacacioglu et al. ${ }^{62}$ found similar CR rates after induction therapy for patients treated with BFM or hyper-CVAD protocols $(95 \%$ and $96 \%$, 
respectively). However, patients in the BFM group showed higher 5-year survival rates compared with the hyper-CVAD group (59\% vs. $34 \%$, respectively). In contrast with these reports, Rytting et al. ${ }^{63}$ found no significant difference in 3 -year OS in a retrospective study of patients treated on an augmented-BFM protocol when compared with historically matched patients treated with hyper-CVAD (74\% and 71\%, respectively). ${ }^{63}$ Outcomes for clinical trials of AYA patients with ALL treated on pediatric-like and hyper-CVAD regimens are summarized in Table $1.50,52,53,55,58-61,63-66$

Similar long-term results were observed in a modeling study of AYA patients treated on hyper-CVAD or a pediatric-inspired protocol. ${ }^{67}$ Investigators used an exploratory decision-analytic approach to model the survival and quality-adjusted survival of AYA patients treated according to these different protocols. Model results at 1 year showed only minor differences between protocols. However, survival and quality-adjusted survival were both noticeably greater in the pediatric-inspired group at 5 and 10 years compared with hyper-CVAD.

\section{Asparaginase}

The use of asparaginase as a chemotherapeutic agent dates back to 1953 when Kidd et al. reported that guinea pig serum induced leukemic cell death when injected into diseased mice. ${ }^{68}$ In 1961, asparaginase was identified as the agent responsible for this antileukemic effect and was subsequently investigated for use in human cancer patients. ${ }^{69}$ Unlike healthy cells, leukemic blasts do not produce asparagine and rely exclusively on extracellular sources of the amino acid. ${ }^{70}$ Asparaginase takes advantage of this metabolic difference by catalyzing the breakdown of asparagine to aspartic acid and ammonia, depriving leukemic cells of the amino acid. ${ }^{71}$ Healthy cells continue to produce asparagine de novo, through the enzymatic action of asparagine synthetase, and are largely spared. However, prolonged asparagine deprivation in leukemic cells results in reduced DNA, RNA, and protein synthesis and eventually leads to the activation of programmed cell death mechanisms. ${ }^{71}$
Asparaginase activity levels show an inverse relationship with circulating asparagine concentrations and are often used to determine a patient's asparagine depletion status. ${ }^{72-75}$ Human and animal studies have shown that serum asparaginase activity levels $\geq 0.1 \mathrm{IU} / \mathrm{mL}$ are adequate for asparagine depletion, and this criterion has become accepted as the minimal therapeutic level in practice. ${ }^{74,76}$ The regular measurement of asparaginase activity was used in several pediatric trials to ensure that the patients maintain asparagine depletion throughout treatment. ${ }^{77,78}$

As a foreign protein, asparaginase has the potential to elicit an immune response when administered to patients. Immune reactions to asparaginase are broadly classified as clinical hypersensitivity or subclinical hypersensitivity (also referred to as "silent inactivation"). Both clinical and subclinical hypersensitivity are associated with reduced asparaginase activity levels in patients and can lead to poor outcomes if not properly addressed. ${ }^{78-81}$

There are three types of asparaginase approved for the treatment of ALL. Native Escherichia coli asparaginase and PEG-asparaginase are derived from the bacterium E. coli. ${ }^{82}$ Owing to their common origins, these asparaginases display a significant amount of cross-reactivity with respect to their ability to elicit an immune response in patients. ${ }^{83}$ The third asparaginase, asparaginase Erwinia chrysanthemi, is derived from the bacterium Erwinia chrysanthemi (revised taxonomy: Dickeya dadantii) and shows limited cross-reactivity with E. coli-derived asparaginases. ${ }^{83,84}$ Asparaginase Erwinia chrysanthemi is indicated as a component of a multiagent chemotherapeutic regimen for the treatment of patients with ALL who have developed hypersensitivity to $E$. coli-derived asparaginase. ${ }^{85}$ Recently, the supply of native E. coli asparaginase has been discontinued in the United States, and has largely been replaced by PEG-asparaginase as first-line treatment for patients with ALL. ${ }^{86}$

All three asparaginases share the same mechanism of action, the deamination of asparagine. However, each asparaginase has markedly different pharmacokinetics, which must be accounted for when establishing dose and treatment schedules (Table 2). ${ }^{87}$ Due to the addition of polyethylene

Table 1. Characteristics and Outcomes in Older Patients Treated on Pediatric-Like AND HyPER-CVAD Protocols FOR ALL

\begin{tabular}{|c|c|c|c|c|c|}
\hline Reference & Patients (n) & Age, years median (range) & $C R$ & OS, years & $O S$ \\
\hline \multicolumn{6}{|l|}{ Pediatric-like } \\
\hline Ribera et al., $2008^{53}$ & 81 & $20.0(15-30)$ & $98 \%$ & 6 & $69 \%$ \\
\hline Huguet et al., $2009^{50}$ & 225 & $31.0(15-60)$ & $93.5 \%$ & 3.5 & $60 \%$ \\
\hline DeAngelo et al., $2007^{64}$ & 75 & $28(18-50)$ & $84 \%$ & 2 & $77 \%$ \\
\hline Rijneveld et al., $2011^{65}$ & 54 & $26(17-40)$ & $91 \%$ & 2 & $72 \%$ \\
\hline Haiat et al., $2011^{52}$ & 40 & $33(18-55)$ & $90 \%$ & 3 & $75 \%$ \\
\hline Cluzeau et al., $2012^{66}$ & 89 & $19(15-29)$ & $99 \%$ & 5 & $66 \%$ \\
\hline Rytting et al., $2014^{63}$ & 85 & $21(13-39)$ & $94 \%$ & 3 & $74 \%$ \\
\hline $\begin{array}{l}\text { Hyper-CVAD } \\
\text { Garcia-Manero et al., } 2000^{59}\end{array}$ & 204 & $39.5(16-79)$ & $91 \%$ & 5 & $<30$ years $=54 \%$ \\
\hline & & & 9170 & $J$ & $30-49$ years $=42 \%$ \\
\hline Kantarjian et al., $2004^{58}$ & 288 & $40(15-92)$ & $92 \%$ & 5 & $38 \%$ \\
\hline Kozlowski et al., $2014^{60}$ & 24 & $32(18-72)$ & $89 \%$ & 5 & $47 \%$ \\
\hline Morris et al., $2011^{55}$ & 63 & $29(14-76)$ & $86 \%$ & 5 & $48 \%$ \\
\hline Buyukasik et al., $2013^{61}$ & 166 & $29(16-63)$ & $84.2 \%$ & 5 & $26.3 \%$ \\
\hline
\end{tabular}

ALL, acute lymphoblastic leukemia; CR, complete response; OS, overall survival. 
Table 2. Half-Life of the Three Asparaginase Preparations

\begin{tabular}{|c|c|c|c|c|}
\hline & $\begin{array}{c}\text { Asparaginase Erwinia } \\
\text { chrysanthemi }{ }^{79} 1 \times i . m . \\
\text { dose of } 25,000 I U / m^{2} \\
(\mathrm{n}=10)\end{array}$ & $\begin{array}{c}\text { Asparaginase } \\
\text { Erwinia chrysanthemi }^{85} \\
1 \times i . v . \text { dose of } \\
25,000 \mathrm{IU} / \mathrm{m}^{2}(\mathrm{n}=24)\end{array}$ & $\begin{array}{l}\text { Native Escherichia } \\
\text { coli asparaginase }{ }^{\mathrm{a}, 79} \\
1 \times \text { i.m. dose of } \\
25,000 \mathrm{IU} / \mathrm{m}^{2}(\mathrm{n}=17)\end{array}$ & $\begin{array}{c}\text { PEG-asparaginase } \\
1 \times \text { i.m. dose of } \\
2500 \mathrm{IU}^{2} \mathrm{~m}^{2}(\mathrm{n}=10)\end{array}$ \\
\hline Half-life, days (mean \pm SD) & $0.65 \pm 0.13$ & $0.31 \pm 1.79$ & $1.28 \pm 0.35$ & $5.73 \pm 3.24$ \\
\hline
\end{tabular}

${ }^{a}$ Native E. coli asparaginase is no longer available in the United States.

i.m., intramuscular; i.v., intravenous; PEG, polyethylene glycol (pegylated); SD, standard deviation.

glycol, PEG-asparaginase shows a longer half-life compared with the other two asparaginase preparations and is therefore able to provide a longer period of asparagine depletion from a single dose. ${ }^{79,88}$ Asparaginase Erwinia chrysanthemi shows a more rapid half-life and should be administered more frequently than PEG-asparaginase to maintain asparagine depletion. ${ }^{87,89}$ The recommended substitution dose of asparaginase Erwinia chrysanthemi in patients who experience hypersensitivity to PEG-asparaginase is $25,000 \mathrm{IU} / \mathrm{m}^{2}$ administered intravenously (i.v.) or intramuscularly (i.m.) six times on a Monday, Wednesday, Friday schedule (2 weeks) to replace each remaining dose of PEG-asparaginase in the patients' scheduled treatment. ${ }^{85,89}$ In 2014, the U.S. Food and Drug Administration approved the i.v. administration of asparaginase Erwinia chrysanthemi ${ }^{85}$ Based on population data, the mean half-life of asparaginase Erwinia chrysanthemi following i.v. infusion was 7.51 hours, compared with 15.6 hours following i.m. injection. ${ }^{85}$ Due to these differences in pharmacokinetics, it is suggested that nadir serum asparaginase activity (NSAA) levels be monitored in patients receiving i.v. asparaginase. Patients may be switched to i.m. administration if desired NSAA levels are not achieved. ${ }^{85}$

\section{Asparaginase Use in AYA Patients}

The pharmacokinetics of asparaginase has been extensively studied in pediatric patients. However, few investigations have focused on asparaginase use in AYA patients. ${ }^{90-92}$ Douer et al. evaluated asparaginase pharmacokinetics in adult patients (aged 17-55 years) with ALL. ${ }^{90}$ Twenty-five newly diagnosed patients were administered a single dose of PEG-asparaginase $2000 \mathrm{IU} / \mathrm{m}^{2}$ during induction. In these adult patients, the population average asparaginase activity correlated well with serum asparagine deamination, and the half-life of i.v. administered PEG-asparaginase was reported as 7 days, similar to what has been shown in pediatric patients. ${ }^{87,92}$ Furthermore, asparaginase activity following PEG-asparaginase administration was long-lasting, with $81 \%$ of patients showing complete deamination of asparagine at 21 days post-infusion.

Although it is not known how long circulating asparagine levels must be depleted for programmed cell death mechanisms to be activated in leukemic blasts, there is strong clinical evidence that prolonged exposure to asparaginase is associated with superior outcomes. ${ }^{93,94}$ The DFCI ALL Consortium Protocol 91-01 evaluated outcomes in 352 pediatric patients (aged 0-18 years) treated on an intensive 30 weeks of high-dose asparaginase during intensification. ${ }^{93}$ The investigators found an increased 5-year EFS in patients who were able to receive $>25$ weeks of asparaginase therapy. EFS was $90 \%$ for patients who received $>25$ weeks of as- paraginase compared with $73 \%$ in patients who tolerated $\leq 25$ weeks $(p<0.01)$. Common reasons for discontinuing asparaginase treatment prior to the 25 -week mark in this study included pancreatitis (39\%), clinical allergy (19\%), and central nervous system thrombosis (12\%).

The CALGB-9511 study measured asparaginase activity in 85 adult patients (aged 17-71 years) receiving PEGasparaginase $2000 \mathrm{IU} / \mathrm{m}^{2}$ during induction and first intensification. ${ }^{91}$ Depletion was defined as trough serum asparaginase activity $>0.03 \mathrm{IU} / \mathrm{mL}$ in this study. The majority of adult patients maintained asparagine depletion $(n=63)$, with 22 patients failing to achieve asparaginase activity $>0.03 \mathrm{IU} / \mathrm{mL}$ in at least one measurement. Adult patients who failed to achieve depletion showed significantly inferior OS and DFS compared with patients with depletion. The reported hazard ratio for OS and DFS between these two groups of patients was $2.37(p=0.002)$ and $2.21(p=0.012)$, respectively.

\section{Safety and Toxicity of Asparaginase in AYA Patients}

Asparaginase use in AYA patients has historically been limited because of the perception of an increased risk of toxicity with age. Hepatotoxicity-grade 3-4 elevations in aspartate aminotransferase and alanine aminotransferase-was the most common toxicity in a study with 152 patients aged 18-60 years given PEG-asparaginase according to a regimen adapted from the augmented arm of the CCG-1882 protocol. $^{95}$ In an evaluation of 76 newly diagnosed adolescent and adult patients (aged 14-68 years) treated with PEG-asparaginase, investigators reported a greater frequency of grade 3-4 hepatic and pancreatic AEs compared with 1274 pediatric patients. ${ }^{96}$ The authors suggest that coexisting morbidities, possibly due to increased use of hepatotoxic and pancreatotoxic drugs, may contribute to the increased toxicities seen in AYA patients, as healthy adults were able to tolerate PEG-asparaginase to an extent comparable with younger patients in the study. ${ }^{96} \mathrm{Al}-$ though increased pancreatic toxicity with asparaginase is often associated with AYA patients, it should be noted that several studies reported a relatively low rate of pancreatitis in AYA patients undergoing asparaginase treatment. ${ }^{50,53,65,95,97}$ Detailed recommendations regarding the management of pancreatitis and other asparaginase-associated toxicities are beyond the scope of this review and have been summarized elsewhere. ${ }^{96}$ The continued use of asparaginase is not recommended in the event of clinically confirmed pancreatitis. ${ }^{96}$

Asparaginase use has also been associated with venous thromboembolic events (VTE) in both pediatric and adult patients. Decreased synthesis of antithrombin and fibrinogen, leading to increased thrombin generation, is commonly associated with asparaginase use. ${ }^{98-100}$ Asparaginase-related VTE has been reported in $3-5 \%$ of pediatric patients undergoing 
treatment for ALL. ${ }^{99,101-104}$ Increased age has been shown to be a significant predictor of VTEs, with thrombotic complications occurring in up to $34 \%$ of adult patients in one study. ${ }^{105}$ Deep venous thrombosis should be managed with anticoagulation therapy, and it is recommended that asparaginase be temporarily discontinued in the case of clinically significant bleeding or thrombotic events. ${ }^{96}$ Many antithrombotic prophylactic approaches have been adopted, including heparin or antithrombin substitution, with varied clinical evidence. ${ }^{102,105,106}$ Asparaginase may be reintroduced once acute toxicity and clinical symptoms have resolved. ${ }^{96}$

Douer et al. ${ }^{107}$ evaluated the pharmacokinetics and toxicity of PEG-asparaginase in 51 adult patients (aged 18-57 years) during induction therapy. In this trial, PEG-asparaginase dosing was structured to avoid overlapping toxicity with other chemotherapy drugs and to improve overall treatment tolerance. Additionally, PEG-asparaginase intervals were lengthened to $\geq 4$ weeks. The investigators reported that the most common asparaginase-associated toxicities (grade 3-4) were hyperbilirubinemia, transaminitis, hyperglycemia, and hypertriglyceridemia (Table 3). No deaths were reported as a result of asparaginase-related toxicity. Overall, PEG-asparaginase was discontinued owing to prohibitive toxicity in $20 \%$ of patients $(n=10)$. The most common reasons for discontinuation in these patients were pancreatitis $(n=6)$ and severe clinical hypersensitivity $(n=3)$.

Clinical hypersensitivity reactions range in severity, from a localized rash or pain around the injection site to a systemic immune reaction, possibly resulting in anaphylaxis. ${ }^{108}$ The prevalence of asparaginase hypersensitivity in pediatric patients with ALL ranges from 3-45\%. ${ }^{75,78,81,97,109,110}$ Although less clinical data exist in adult patients, hypersensitivity rates do not appear to differ with age. In CALGB-8811, the incidence of severe hypersensitivity reactions was reported as $11 \%$ for adults treated with native $E$. coli asparaginase. ${ }^{111}$ For newly diagnosed adult patients (aged 15-39 years) enrolled in the U.S. Intergroup Trial C10403, grade 3-4 hypersensitivity

Table 3. PEG-Asparaginase Toxicity in Patients Aged 18-57 Years $(N=51)^{107}$

\begin{tabular}{|c|c|c|c|}
\hline \multirow[b]{2}{*}{ Toxicity } & \multirow{2}{*}{$\frac{\text { Grade } 1 \text { or } 2}{\mathrm{n}}$} & \multicolumn{2}{|c|}{ Grade 3 or 4} \\
\hline & & $\mathrm{n}$ & $\%$ \\
\hline Pancreatitis & 0 & 7 & 13.7 \\
\hline Allergy & 2 & 3 & 5.9 \\
\hline Deep venous thrombosis & 0 & 8 & 15.7 \\
\hline Bleeding & 5 & 1 & 2 \\
\hline Transaminitis & 19 & 32 & 62.7 \\
\hline Hyperbilirubinemia & 30 & 16 & 31.3 \\
\hline Hyperglycemia & 29 & 17 & 33.3 \\
\hline Hypertriglyceridemia & 6 & 9 & 17.6 \\
\hline Fatigue & 34 & 4 & 7.8 \\
\hline Neuropathy & 10 & 1 & 2 \\
\hline Vomiting & 19 & 2 & 3.9 \\
\hline Nausea & 34 & 3 & 5.9 \\
\hline Diarrhea & 16 & 0 & 0 \\
\hline Constipation & 18 & 0 & 0 \\
\hline Headache & 27 & 1 & 2 \\
\hline Edema & 6 & 0 & 0 \\
\hline
\end{tabular}

Reprinted and adapted with permission from Douer et al. 2014. ${ }^{107}$ (C) 2014 American Society of Clinical Oncology. All rights reserved. reactions were found to occur in $8-13 \%$ of patients treated with PEG-asparaginase. ${ }^{12,113}$ In the compassionate-use trial for asparaginase Erwinia chrysanthemi, hypersensitivity reactions were reported in $11 \%$ of AYA patients compared with $15 \%$ of patients aged 10 years and younger. ${ }^{97}$ Patients with hypersensitivity to $E$. coli-derived asparaginase should immediately discontinue therapy and be switched to treatment with asparaginase Erwinia chrysanthemi. Asparaginase Erwinia chrysanthemi is indicated for use as a component of a multiagent chemotherapeutic regimen for the treatment of patients with ALL who have developed hypersensitivity to E. coli-derived asparaginase. ${ }^{80,82}$

The development of antiasparaginase antibodies can occur in patients without any overt signs of an immune response, a condition referred to as subclinical hypersensitivity or "silent inactivation." $78,81,114$ Subclinical hypersensitivity in pediatric patients is characterized by decreased asparaginase activity levels and can be associated with poor outcomes in those who have not switched to an alternative asparagine preparation once subclinical hypersensitivity is established. ${ }^{77,78,81}$ Asparaginase Erwinia chrysanthemi shows no cross-reactivity with $E$. coliderived asparaginases and is the preferred alternative in patients who develop allergy to native $E$. coli or PEGasparaginase. ${ }^{76,83,96}$ The regular monitoring of asparaginase activity levels throughout the course of therapy can help identifiy patients who develop subclinical hypersensitivity and allows clinicians to adjust treatment actively to the individual patient. ${ }^{78}$ In a study by Burke et al., patients aged 18-57 years were treated with PEG-asparaginase according to a regimen adapted from the pediatric protocol CCG-1882. ${ }^{115}$ A total of 34 of 61 PEG-asparaginase doses were associated with antiasparagainse antibody production, 27 of which occurred within the first 7 days post-dose, and only two of which were associated with an overt hypersensitivity response. However, serum asparaginase activity $<0.2 \mathrm{IU} / \mathrm{mL}$ at 14 days post-dose was found in only 2 of 26 antibodypositive patients, suggesting that antibody production is not associated with increased drug clearance in these patients.

A large $(n=1368)$ compassionate-use trial evaluated the safety and toxicity of asparaginase Erwinia chrysanthemi in patients of various ages. ${ }^{97}$ The study included 147 AYA patients (aged 16-39 years) and reported that the safety profile of asparaginase Erwinia chrysanthemi in these patients was consistent with that found in the total population (Fig. 2). The reported incidence of pancreatitis was similar for patients aged 16-40 years and younger than 10 years $(3.4 \%$ and $3.0 \%$, respectively). Rates of thrombosis were slightly higher in AYA patients, with thrombosis reported in $4.1 \%$ of AYA patients compared with $1.3 \%$ of children younger than 10 years of age. All patients included in the study had a previous grade $\geq 2$ hypersensitivity reaction to $E$. coli-derived asparaginase and were switched to asparaginase Erwinia chrysanthemi $25,000 \mathrm{IU} / \mathrm{m}^{2}$ administered on a Monday, Wednesday, Friday regimen. Overall, asparaginase Erwinia chrysanthemi was well-tolerated in AYA patients and allowed the majority (72.8\%) to complete their planned course of asparaginase treatment. Although outcomes were not reported in this study, the therapeutic benefits of receiving the full course of asparaginase treatment are well-established. ${ }^{97}$

Safety and toxicity data from a large prospective trial evaluating AYA patients treated on a pediatric regimen were recently presented in abstract form. ${ }^{12,113}$ Between November 


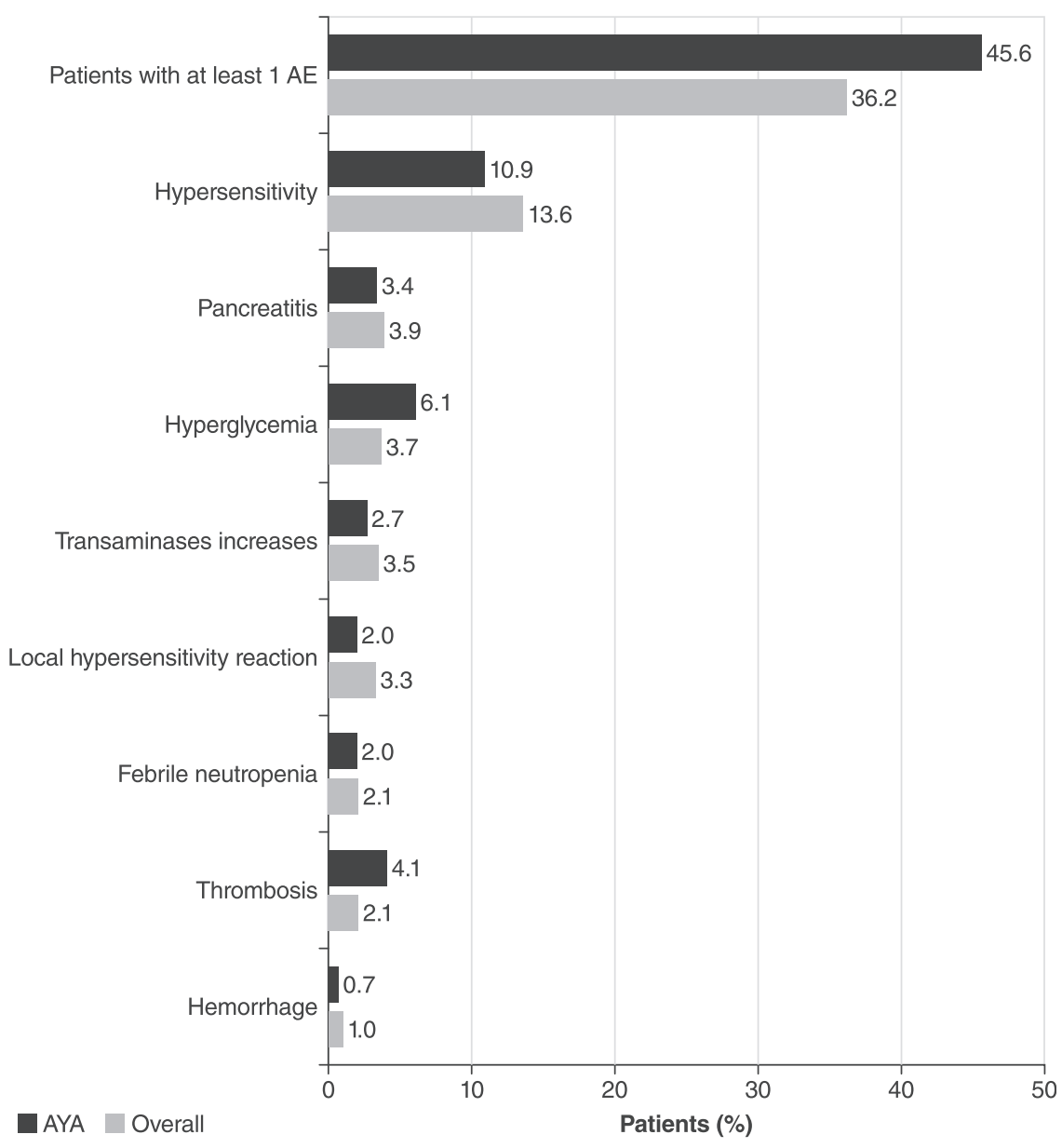

FIG. 2. Comparison of adverse events (AEs) in AYAs $(n=147)$ with the overall patient population $(n=940)$ in a compassionate-use trial of asparaginase Erwinia chrysanthemi. ${ }^{9}$
2007 and August 2012, 318 patients (aged 16-39 years) were enrolled in the U.S. Intergroup Trial C10403 and treated according to the Children's Oncology Group (COG AALL0232) protocol by adult oncologists. Safety and toxicity results were compared with 159 patients from COG AALL0232. The incidence of hepatic toxicity, pancreatitis, and osteonecrosis were found to be similar between AYA patients in both groups. Grades 3-4 hepatic and pancreatic dysfunction were slightly greater in C10403 compared with pediatric patients, likely due to an increased use of alcohol or other hepatotoxic and pancreatotoxic drugs. Overall, healthy adults were able to tolerate asparaginase therapy comparable with younger patients. Treatment-related mortality was low (2\%) in C10403, and the investigators concluded that treatment with a pediatric regimen was feasible in AYA patients up to 40 years of age.

\section{Conclusions and Future Directions}

The treatment of AYA patients diagnosed with ALL represents a unique challenge to the clinical oncologist. Substantial evidence indicates that AYA patients treated on pediatric protocols show improved survival compared with patients treated on traditional adult regimens. Although certain treatment-related toxicities are more prevalent in AYA patients, emerging clinical evidence suggests that highintensity pediatric regimens are feasible in the AYA population. As is the case in pediatrics, the effective management of treatment-related toxicities is critical to ensure that AYA patients receive the full benefit of ALL therapy. The ongoing development of novel asparaginase preparations, such as pegylated recombinant Erwinia-derived asparaginase (pegcrisantaspase) and red blood-cell encapsulated asparaginase, also promises to reduce immunogenicity and increase the overall length of asparagine depletion.

\section{Acknowledgments}

The authors wish to thank Cory Hussar, PhD, of The Curry Rockefeller Group, LLC, Tarrytown, NY, for providing editorial assistance that was supported by Jazz Pharmaceuticals plc or its subsidiaries.

\section{Author Disclosure Statement}

Leonard Sender is on the speakers' bureau of Jazz Pharmaceuticals and is involved in scientific research funded by Jazz Pharmaceuticals or its subsidiaries.

No competing financial interests exist for Nicolas Boissel.

\section{References}

1. Inaba H, Greaves M, Mullighan CG. Acute lymphoblastic leukaemia. Lancet. 2013;381(9881):1943-55.

2. Siegel R, Naishadham D, Jemal A. Cancer statistics, 2012. CA Cancer J Clin. 2012;62(1):10-29.

3. Pui $\mathrm{CH}$, Robison LL, Look AT. Acute lymphoblastic leukaemia. Lancet. 2008;371(9617):1030-43.

4. Pulte D, Gondos A, Brenner H. Trends in 5- and 10-year survival after diagnosis with childhood hematologic 
malignancies in the United States, 1990-2004. J Natl Cancer Inst. 2008;100(18):1301-9.

5. Pui $\mathrm{CH}$. Recent research advances in childhood acute lymphoblastic leukemia. J Formos Med Assoc. 2010; 109(11):777-87.

6. Hunger SP, Lu X, Devidas M, et al. Improved survival for children and adolescents with acute lymphoblastic leukemia between 1990 and 2005: a report from the Children's Oncology Group. J Clin Oncol. 2012;30(14):1663-9.

7. Pulte D, Gondos A, Brenner H. Improvement in survival in younger patients with acute lymphoblastic leukemia from the 1980 s to the early 21 st century. Blood. 2009; 113(7):1408-11.

8. Faderl S, Jeha S, Kantarjian HM. The biology and therapy of adult acute lymphoblastic leukemia. Cancer. 2003; 98(7):1337-54.

9. Nachman J. Clinical characteristics, biologic features and outcome for young adult patients with acute lymphoblastic leukaemia. Br J Haematol. 2005;130(2):166-73.

10. Schafer ES, Hunger SP. Optimal therapy for acute lymphoblastic leukemia in adolescents and young adults. Nat Rev Clin Oncol. 2011;8(7):417-24.

11. Douer D. Is asparaginase a critical component in the treatment of acute lymphoblastic leukemia? Best Pract Res Clin Haematol. 2008;21(4):647-58.

12. Boissel N, Huguet F, Dombret H. Treatment of acute lymphoblastic leukemia in young adults. In: Adult acute lymphocytic leukemia. Advani AS, Lazarus HM (Eds). New York: Springer; 2011; pp. 211-32.

13. Kantarjian HM, O'Brien S, Smith TL, et al. Results of treatment with hyper-CVAD, a dose-intensive regimen, in adult acute lymphocytic leukemia. J Clin Oncol. 2000; 18(3):547-61.

14. Dombret H, Cluzeau T, Huguet F, Boissel N. Pediatriclike therapy for adults with ALL. Curr Hematol Malig Rep. 2014;9(2):158-64.

15. Boissel N, Auclerc MF, Lhéritier V, et al. Should adolescents with acute lymphoblastic leukemia be treated as old children or young adults? Comparison of the French FRALLE-93 and LALA-94 trials. J Clin Oncol. 2003;21(5):774-80.

16. de Bont JM, Holt B, Dekker AW, et al. Significant difference in outcome for adolescents with acute lymphoblastic leukemia treated on pediatric vs adult protocols in the Netherlands. Leukemia. 2004;18(12):2032-5.

17. Hallbook H, Gustafsson G, Smedmyr B, et al. Treatment outcome in young adults and children $>10$ years of age with acute lymphoblastic leukemia in Sweden: a comparison between a pediatric protocol and an adult protocol. Cancer. 2006;107(7):1551-61.

18. Ramanujachar R, Richards S, Hann I, et al. Adolescents with acute lymphoblastic leukaemia: outcome on UK national paediatric (ALL97) and adult (UKALLXII/ E2993) trials. Pediatr Blood Cancer. 2007;48(3):254-61.

19. Stock W, La M, Sanford B, et al. What determines the outcomes for adolescents and young adults with acute lymphoblastic leukemia treated on cooperative group protocols? A comparison of Children's Cancer Group and Cancer and Leukemia Group B studies. Blood. 2008; 112(5):1646-54.

20. Usvasalo A, Raty R, Knuutila S, et al. Acute lymphoblastic leukemia in adolescents and young adults in Finland. Haematologica. 2008;93(8):1161-8.

21. Testi AM, Valsecchi MG, Conter V, et al. Difference in outcome of adolescents with acute lymphoblastic leuke- mia (ALL) enrolled in pediatric (AIEOP) and adult (GIMEMA) protocols. Blood. 2004;104(11):abstract 1954.

22. Ramanujachar R, Richards S, Hann I, Webb D. Adolescents with acute lymphoblastic leukaemia: emerging from the shadow of paediatric and adult treatment protocols. Pediatr Blood Cancer. 2006;47(6):748-56.

23. Ribera JM, Oriol A. Acute lymphoblastic leukemia in adolescents and young adults. Hematol Oncol Clin North Am. 2009;23(5):1033-42, vi.

24. McNeer JL, Raetz EA. Acute lymphoblastic leukemia in young adults: which treatment? Curr Opin Oncol. 2012; 24(5):487-94.

25. Uckun FM, Reaman G, Steinherez PG, et al. Improved clinical outcome for children with T-lineage acute lymphoblastic leukemia after contemporary chemotherapy: a Children's Cancer Group Study. Leuk Lymphoma. 1996; 24(1-2):57-70.

26. Ma M, Wang X, Tang J, et al. Early T-cell precursor leukemia: a subtype of high risk childhood acute lymphoblastic leukemia. Front Med. 2012;6(4):416-20.

27. Coustan-Smith E, Mullighan CG, Onciu M, et al. Early Tcell precursor leukaemia: a subtype of very high-risk acute lymphoblastic leukaemia. Lancet Oncol. 2009;10(2):147-56.

28. Wood BL, Winter SS, Dunsmore KP, et al. T-lymphoblastic leukemia (T-ALL) shows excellent outcome, lack of significance of the early thymic precursor (ETP) immunophenotype, and validation of the prognostic value of endinduction minimal residual disease (MRD) in Children's Oncology Group (COG) study AALL0434. Blood. 2014; 124(21):abstract.

29. Harrison CJ. Cytogenetics of paediatric and adolescent acute lymphoblastic leukaemia. Br J Haematol. 2009;144(2): 147-56.

30. Faderl S, Kantarjian HM, Talpaz M, Estrov Z. Clinical significance of cytogenetic abnormalities in adult acute lymphoblastic leukemia. Blood. 1998;91(11):3995-4019.

31. Secker-Walker LM, Craig JM, Hawkins JM, Hoffbrand AV. Philadelphia positive acute lymphoblastic leukemia in adults: age distribution, BCR breakpoint and prognostic significance. Leukemia. 1991;5(3):196-9.

32. Aguiar RC, Sohal J, van Rhee F, et al. TEL-AML1 fusion in acute lymphoblastic leukaemia of adults. Br J Haematol. 1996;95(4):673-7.

33. Roberts KG, Payne-Turner D, Pei D, et al. Integrated genomic and mutational profiling of adolescent and young adult ALL identifies a high frequency of BCR-ABL1-Like ALL with very poor outcome. Blood. 2013;122(21): abstract 825 .

34. Vey N, Thomas X, Picard C, et al. Allogeneic stem cell transplantation improves the outcome of adults with $\mathrm{t}(1 ; 19) / E 2 A-P B X 1$ and $\mathrm{t}(4 ; 11) / M L L-A F 4$ positive B-cell acute lymphoblastic leukemia: results of the prospective multicenter LALA-94 study. Leukemia. 2006;20(12): 2155-61.

35. Den Boer ML, van Slegtenhorst M, De Menezes RX, et al. A subtype of childhood acute lymphoblastic leukaemia with poor treatment outcome: a genome-wide classification study. Lancet Oncol. 2009;10(2):125-34.

36. Roberts KG, Li Y, Payne-Turner D, Harvey RC, et al. Targetable kinase-activating lesions in Ph-like acute lymphoblastic leukemia. N Engl J Med. 2014;371(11): 1005-15.

37. Roberts KG, Pei D, Campana D, et al. Outcomes of children with BCR-ABL1-like acute lymphoblastic leukemia 
treated with risk-directed therapy based on the levels of minimal residual disease. J Clin Oncol. 2014;32(27): 3012-20.

38. Pieters R, den Boer ML, Durian M, et al. Relation between age, immunophenotype and in vitro drug resistance in 395 children with acute lymphoblastic leukemia-implications for treatment of infants. Leukemia. 1998;12(9):1344-8.

39. Fernandez CA, Smith C, Yang W, et al. Genome-wide analysis links NFATC2 with asparaginase hypersensitivity. Blood. 2015;126(1):69-75.

40. Yang L, Panetta JC, Cai X, et al. Asparaginase may influence dexamethasone pharmacokinetics in acute lymphoblastic leukemia. J Clin Oncol. 2008;26(12):1932-9.

41. Meadows AT, Kramer S, Hopson R, et al. Survival in childhood acute lymphocytic leukemia: effect of protocol and place of treatment. Cancer Invest. 1983;1(1):49-55.

42. Stiller CA, Eatock EM. Patterns of care and survival for children with acute lymphoblastic leukaemia diagnosed between 1980 and 1994. Arch Dis Child. 1999;81(3):202-8.

43. Fern LA, Lewandowski JA, Coxon KM, Whelan J. Available, accessible, aware, appropriate, and acceptable: a strategy to improve participation of teenagers and young adults in cancer trials. Lancet Oncol. 2014;15(8):e341-50.

44. Fern LA, Whelan JS. Recruitment of adolescents and young adults to cancer clinical trials - international comparisons, barriers, and implications. Semin Oncol. 2010;37(2):e1-8.

45. Jeha S. Who should be treating adolescents and young adults with acute lymphoblastic leukaemia? Eur J Cancer. 2003;39(18):2579-83.

46. de Oliveira BM, Viana MB, Zani CL, Romanha AJ. Clinical and laboratory evaluation of compliance in acute lymphoblastic leukaemia. Arch Dis Child. 2004;89(8):785-8.

47. Bhatia S, Landier W, Shangguan M, et al. Nonadherence to oral mercaptopurine and risk of relapse in Hispanic and non-Hispanic white children with acute lymphoblastic leukemia: a report from the Children's Oncology Group. J Clin Oncol. 2012;30(17):2094-101.

48. Goldstone AH, Richards SM, Lazarus HM, et al. In adults with standard-risk acute lymphoblastic leukemia, the greatest benefit is achieved from a matched sibling allogeneic transplantation in first complete remission, and an autologous transplantation is less effective than conventional consolidation/maintenance chemotherapy in all patients: final results of the International ALL Trial (MRC UKALL XII/ECOG E2993). Blood. 2008;111(4):1827-33.

49. Peters C, Schrauder A, Schrappe M, et al. Allogeneic haematopoietic stem cell transplantation in children with acute lymphoblastic leukaemia: the BFM/IBFM/EBMT concepts. Bone Marrow Transplant. 2005;35(1 Suppl):S9-11.

50. Huguet F, Leguay T, Raffoux E, et al. Pediatric-inspired therapy in adults with Philadelphia chromosome-negative acute lymphoblastic leukemia: the GRAALL-2003 study. J Clin Oncol. 2009;27(6):911-18.

51. Storring JM, Minden MD, Kao S, et al. Treatment of adults with BCR-ABL negative acute lymphoblastic leukaemia with a modified paediatric regimen. Br J Haematol. $2009 ; 146(1): 76-85$.

52. Haiat S, Marjanovic Z, Lapusan S, et al. Outcome of 40 adults aged from 18 to 55 years with acute lymphoblastic leukemia treated with double-delayed intensification pediatric protocol. Leuk Res. 2011;35(1):66-72.

53. Ribera JM, Oriol A, Sanz MA, et al. Comparison of the results of the treatment of adolescents and young adults with standard-risk acute lymphoblastic leukemia with the
Programa Espanol de Tratamiento en Hematología pediatric-based protocol ALL-96. J Clin Oncol. 2008; 26(11):1843-9.

54. Fathi AT, DeAngelo DJ, Stevenson KE, et al. Intensified chemotherapy for older patients with acute lymphoblastic leukemia (ALL): a phase II study from the Dana Farber Cancer Institute (DFCI) ALL Consortium. Blood. 2014; 124(21):abstract 3714.

55. Morris K, Weston H, Mollee P, et al. Outcome of treatment of adult acute lymphoblastic leukemia with hyperfractionated cyclophosphamide, doxorubicin, vincristine, dexamethasone/methotrexate, cytarabine: results from an Australian population. Leuk Lymphoma. 2011;52(1):85-91.

56. Toft N, Schmiegelow K, Klausen TW, Birgens H. Adult acute lymphoblastic leukaemia in Denmark. A national population-based retrospective study on acute lymphoblastic leukaemia in Denmark 1998-2008. Br J Haematol. 2012;157(1):97-104.

57. Faderl S, Thomas DA, O'Brien S, et al. Augmented hyperCVAD based on dose-intensified vincristine, dexamethasone, and asparaginase in adult acute lymphoblastic leukemia salvage therapy. Clin Lymphoma Myeloma Leuk. 2011;11(1):54-9.

58. Kantarjian H, Thomas D, O'Brien S, et al. Long-term follow-up results of hyperfractionated cyclophosphamide, vincristine, doxorubicin, and dexamethasone (Hyper-CVAD), a dose-intensive regimen, in adult acute lymphocytic leukemia. Cancer. 2004;101(12):2788-801.

59. Garcia-Manero G, Kantarjian HM. The hyper-CVAD regimen in adult acute lymphocytic leukemia. Hematol Oncol Clin North Am. 2000;14(6):1381-96, x-xi.

60. Kozlowski P, Astrom M, Ahlberg L, et al. High relapse rate of $\mathrm{T}$ cell acute lymphoblastic leukemia in adults treated with Hyper-CVAD chemotherapy in Sweden. Eur J Haematol. 2014;92(5):377-81.

61. Buyukasik Y, Acar K, Kelkitli E, et al. Hyper-CVAD regimen in routine management of adult acute lymphoblastic leukemia: a retrospective multicenter study. Acta Haematol. 2013;130(3):199-205.

62. Alacacioglu I, Medeni SS, Ozsan GH, et al. Is the BFM regimen feasible for the treatment of adult acute lymphoblastic leukemia? A retrospective analysis of the outcomes of BFM and Hyper-CVAD chemotherapy in two centers. Chemotherapy. 2014;60(4):219-23.

63. Rytting ME, Thomas DA, O'Brien SM, et al. Augmented Berlin-Frankfurt-Münster therapy in adolescents and young adults (AYAs) with acute lymphoblastic leukemia (ALL). Cancer. 2014;120(23):3660-8.

64. DeAngelo DJ, Dahlberg S, Silverman LB, et al. A multicenter phase II study using a dose intensified pediatric regimen in adults with untreated acute lymphoblastic leukemia. Blood. 2007;110(11):abstract 587.

65. Rijneveld AW, van der Holt B, Daenen SM, et al. Intensified chemotherapy inspired by a pediatric regimen combined with allogeneic transplantation in adult patients with acute lymphoblastic leukemia up to the age of 40 . Leukemia. 2011;25(11):1697-703.

66. Cluzeau N, Dhedin N, Huguet F, et al. Dose-intensity impacts on survival of adolescents and young adults with acute lymphoblastic leukemia treated in adult departments by a pediatric protocol (FRALLE 2000BT). Blood. 2012;120(21):abstract 3561.

67. Guzauskas GF, Villa KF, Vanhove TF, Veenstra DL. Riskbenefit trade-off of pediatric-inspired versus hyper-CVAD 
protocols for Philadelphia-negative acute lymphocytic leukemia (ALL) in adolescents and young adults: a modeling analysis. Blood. 2014;124(21):abstract 1281.

68. Kidd JG. Regression of transplanted lymphomas induced in vivo by means of normal guinea pig serum. I. Course of transplanted cancers of various kinds in mice and rats given guinea pig serum, horse serum, or rabbit serum. J Exp Med. 1953;98(6):565-82.

69. Broome JD. Evidence that the L-asparaginase activity of guinea pig serum is responsible for its antilymphoma effects. Nature. 1961;191:1114-15.

70. Cooney DA, Handschumacher RE. L-asparaginase and L-asparagine metabolism. Annu Rev Pharmacol. 1970;10: 421-40.

71. Nandy P, Periclou AP, Avramis VI. The synergism of 6mercaptopurine plus cytosine arabinoside followed by PEG-asparaginase in human leukemia cell lines (CCRF/ $\mathrm{CEM} / 0$ and (CCRF/CEM/ara-C/7A) is due to increased cellular apoptosis. Anticancer Res. 1998;18(2A):727-37.

72. Boos J, Werber G, Ahlke E, et al. Monitoring of asparaginase activity and asparagine levels in children on different asparaginase preparations. Eur J Cancer. 1996;32A(9):1544-50.

73. Grigoryan RS, Panosyan EH, Seibel NL, et al. Changes of amino acid serum levels in pediatric patients with higherrisk acute lymphoblastic leukemia (CCG-1961). In Vivo. 2004;18(2):107-12.

74. Riccardi R, Holcenberg JS, Glaubiger DL, et al. L-asparaginase pharmacokinetics and asparagine levels in cerebrospinal fluid of rhesus monkeys and humans. Cancer Res. 1981;41(11 Pt 1):4554-8.

75. Avramis VI, Sencer S, Periclou AP, et al. A randomized comparison of native Escherichia coli asparaginase and polyethylene glycol conjugated asparaginase for treatment of children with newly diagnosed standard-risk acute lymphoblastic leukemia: a Children's Cancer Group study. Blood. 2002;99(6):1986-94.

76. Pieters R, Hunger SP, Boos J, et al. L-asparaginase treatment in acute lymphoblastic leukemia: a focus on Erwinia asparaginase. Cancer. 2011;117(2):238-49.

77. Tong WH, Pieters R, Kaspers GJ, et al. A prospective study on drug monitoring of PEGasparaginase and Erwinia asparaginase and asparaginase antibodies in pediatric acute lymphoblastic leukemia. Blood. 2014;123(13): 2026-33.

78. Vrooman LM, Stevenson KE, Supko JG, et al. Postinduction dexamethasone and individualized dosing of Escherichia coli L-asparaginase each improve outcome of children and adolescents with newly diagnosed acute lymphoblastic leukemia: results from a randomized studyDana-Farber Cancer Institute ALL Consortium Protocol 00-01. J Clin Oncol. 2013;31(9):1202-10.

79. Asselin BL, Whitin JC, Coppola DJ, et al. Comparative pharmacokinetic studies of three asparaginase preparations. J Clin Oncol. 1993;11(9):1780-6.

80. Zalewska-Szewczyk B, Andrzejewski W, Mlynarski W, et al. The anti-asparagines antibodies correlate with Lasparagines activity and may affect clinical outcome of childhood acute lymphoblastic leukemia. Leuk Lymphoma. 2007;48(5):931-6.

81. Panosyan EH, Seibel NL, Martin-Aragon S, et al. Asparaginase antibody and asparaginase activity in children with higher-risk acute lymphoblastic leukemia: Children's Cancer Group Study CCG-1961. J Pediatr Hematol Oncol. 2004;26(4):217-26.
82. Panetta JC, Gajjar A, Hijiya N, et al. Comparison of native E. coli and PEG asparaginase pharmacokinetics and pharmacodynamics in pediatric acute lymphoblastic leukemia. Clin Pharmacol Ther. 2009;86(6):651-8.

83. Zalewska-Szewczyk B, Gach A, Wyka K, et al. The crossreactivity of anti-asparaginase antibodies against different $\mathrm{L}$ asparaginase preparations. Clin Exp Med. 2009;9(2):113-16.

84. Samson R, Legendre JB, Christen R, et al. Transfer of Pectobacterium chrysanthemi (Burkholder et al. 1953) Brenner et al. 1973 and Brenneria paradisiaca to the genus Dickeya gen. nov. as Dickeya chrysanthemi comb. nov. and Dickeya paradisiaca comb. nov. and delineation of four novel species, Dickeya dadantii sp. nov., Dickeya dianthicola sp. nov., Dickeya dieffenbachiae sp. nov. and Dickeya zeae sp. nov. Int J Syst Evol Microbiol. 2005; 55(Pt 4):1415-27.

85. ERWINAZE ${ }^{\circledR}$ [package insert]. Palo Alto, CA: Jazz Pharmaceuticals, Inc.; December 2014. Accessed: January 30, 2015 from: http://erwinaze.com/ERWINAZEPI.pdf

86. Keding R. RE: Discontinuation of Elspar ${ }^{\circledR}$, (asparaginase for injection) 10,000 IU. Accessed July 30, 2014 from: http:// www.fda.gov/downloads/Drugs/DrugSafety/DrugShortages/ UCM321556.pdf

87. Asselin BL. The three asparaginases. Comparative pharmacology and optimal use in childhood leukemia. Adv Exp Med Biol. 1999;457:621-9.

88. Dinndorf PA, Gootenberg J, Cohen MH, et al. FDA drug approval summary: pegaspargase $\left(\right.$ Oncaspar $\left.^{\circledR}\right)$ for the first-line treatment of children with acute lymphoblastic leukemia (ALL). Oncologist. 2007;12(8):991-8.

89. Salzer WL, Asselin B, Supko JG, et al. Erwinia asparaginase achieves therapeutic activity after pegaspargase allergy: a report from the Children's Oncology Group. Blood. 2013;122(4):507-14.

90. Douer D, Yampolsky H, Cohen LJ, et al. Pharmacodynamics and safety of intravenous pegaspargase during remission induction in adults aged 55 years or younger with newly diagnosed acute lymphoblastic leukemia. Blood. 2007;109(7):2744-50.

91. Wetzler M, Sanford BL, Kurtzberg J, et al. Effective asparagine depletion with pegylated asparaginase results in improved outcomes in adult acute lymphoblastic leukemia: Cancer and Leukemia Group B Study 9511. Blood. 2007;109(10):4164-7.

92. Avramis VI, Panosyan EH. Pharmacokinetic/pharmacodynamic relationships of asparaginase formulations. Clin Pharmacokinet. 2005;44(4):367-93.

93. Silverman LB, Gelber RD, Dalton VK, et al. Improved outcome for children with acute lymphoblastic leukemia: results of Dana-Farber Consortium Protocol 91-01. Blood. 2001;97(5):1211-18.

94. Pession A, Valsecchi MG, Masera G, et al. Long-term results of a randomized trial on extended use of high dose L-asparaginase for standard risk childhood acute lymphoblastic leukemia. J Clin Oncol. 2005;23(28): 7161-7.

95. Aldoss I, Pullarkat V, Chaudhary P, Douer D. The toxicity profile of repeated dosing of peg-asparaginase in 152 adults with acute lymphoblastic leukemia. Blood. 2014; 124(11):abstract 2294.

96. Stock W, Douer D, DeAngelo DJ, et al. Prevention and management of asparaginase/pegasparaginase-associated toxicities in adults and older adolescents: recommendations of an expert panel. Leuk Lymphoma. 2011;52(12):2237-53. 
97. Plourde PV, Jeha S, Hijiya N, et al. Safety profile of asparaginase Erwinia chrysanthemi in a large compassionateuse trial. Pediatr Blood Cancer. 2014;61(7):1232-8.

98. Hongo T, Okada S, Ohzeki T, et al. Low plasma levels of hemostatic proteins during the induction phase in children with acute lymphoblastic leukemia: a retrospective study by the JACLS. Pediatr Int. 2002;44(3):293-9.

99. Mitchell L, Hoogendoorn H, Giles AR, et al. Increased endogenous thrombin generation in children with acute lymphoblastic leukemia: risk of thrombotic complications in L'Asparaginase-induced antithrombin III deficiency. Blood. 1994;83(2):386-91.

100. Andrew M, Brooker L, Mitchell L. Acquired antithrombin III deficiency secondary to asparaginase therapy in childhood acute lymphoblastic leukaemia. Blood Coagul Fibrinolysis. 1994;5(1 Suppl):S24-36.

101. Caruso V, Iacoviello L, Di Castelnuovo A, et al. Thrombotic complications in childhood acute lymphoblastic leukemia: a meta-analysis of 17 prospective studies comprising 1752 pediatric patients. Blood. 2006;108(7):2216-22.

102. Payne JH, Vora AJ. Thrombosis and acute lymphoblastic leukaemia. Br J Haematol. 2007;138(4):430-45.

103. Nowak-Gottl U, Kenet G, Mitchell LG. Thrombosis in childhood acute lymphoblastic leukaemia: epidemiology, aetiology, diagnosis, prevention and treatment. Best Pract Res Clin Haematol. 2009;22(1):103-14.

104. Qureshi A, Mitchell C, Richards S, et al. Asparaginaserelated venous thrombosis in UKALL 2003- re-exposure to asparaginase is feasible and safe. Br J Haematol. 2010; 149(3):410-13.

105. Grace RF, Dahlberg SE, Neuberg D, et al. The frequency and management of asparaginase-related thrombosis in paediatric and adult patients with acute lymphoblastic leukaemia treated on Dana-Farber Cancer Institute consortium protocols. Br J Haematol. 2011;152(4):452-9.

106. Truelove E, Fielding AK, Hunt BJ. The coagulopathy and thrombotic risk associated with L-asparaginase treatment in adults with acute lymphoblastic leukaemia. Leukemia. 2013;27(3):553-9.

107. Douer D, Aldoss I, Lunning MA, et al. Pharmacokineticsbased integration of multiple doses of intravenous pegaspargase in a pediatric regimen for adults with newly diagnosed acute lymphoblastic leukemia. J Clin Oncol. 2014;32(9):905-11.
108. Shinnick SE, Browning ML, Koontz SE. Managing hypersensitivity to asparaginase in pediatrics, adolescents, and young adults. J Pediatr Oncol Nurs. 2013;30(2): 63-77.

109. Woo MH, Hak LJ, Storm MC, et al. Hypersensitivity or development of antibodies to asparaginase does not impact treatment outcome of childhood acute lymphoblastic leukemia. J Clin Oncol. 2000;18(7):1525-32.

110. Muller HJ, Beier R, Loning L, et al. Pharmacokinetics of native Escherichia coli asparaginase (Asparaginase medac) and hypersensitivity reactions in ALL-BFM 95 reinduction treatment. Br J Haematol. 2001;114(4):794-9.

111. Larson RA, Fretzin MH, Dodge RK, Schiffer CA. Hypersensitivity reactions to L-asparaginase do not impact on the remission duration of adults with acute lymphoblastic leukemia. Leukemia. 1998;12(5):660-5.

112. Advani AS, Sanford B, Luger S, et al. Frontline-treatment of acute lymphoblastic leukemia (ALL) in older adolescents and young adults (AYA) using a pediatric regimen is feasible: toxicity results of the prospective US Intergroup Trial C10403 (Alliance). Blood. 2013;122(21):abstract 3903.

113. Stock W, Luger SM, Advani AS, et al. Favorable outcomes for older adolescents and young adults (AYA) with acute lymphoblastic leukemia (ALL): early results of US Intergroup Trial C10403. Blood. 2014;124(21):abstract 796.

114. Liu C, Kawedia JD, Cheng C, et al. Clinical utility and implications of asparaginase antibodies in acute lymphoblastic leukemia. Leukemia. 2012;26(11):2303-9.

115. Burke PW, Aldoss I, Ramezani L, et al. Frequency and characteristics of pegaspargase antibodies in adults treated with a pediatric acute lymphoblastic leukemia regimen. Blood. 2014;124(21):abstract 936.

Address correspondence to: Leonard S. Sender, MD

Director, Clinical Oncology Services Department of Medicine

Department of Hematology/Oncology University of California, Irvine

Building 56, Room 205 Irvine, $C A 92697$

Email: 1sender@uci.edu 